\title{
( The Use of Emoji in International Communication: aße A Cultural Bridge for Businesses or a Potential ResearchHouse Pitfall
}

\section{David E Missirian}

Department of Law, Taxation and Financial Planning, Bentley University, Waltham, MA 02452, USA

E-mail for correspondence: $\underline{\text { dmissirian@bentley.edu }}$

\begin{abstract}
There is a new form of communication entering the international business world and the legal environment. It is the Emoji. It has been embraced across the globe by millennials. There are 26 million US persons entering the business community this year alone for whom the emoji is an essential communications tool. That number is matched in multiples by other countries. We must anticipate and incorporate this new mode and method of communication into our international legal structure, or we will ultimately make our legal system less effective in the global business community driving a communications wedge between our society and the rest of the world.
\end{abstract}

Keywords: commerce, emoji, communication, international business

\section{INTRODUCTION}

This paper examines the meteoric rise of a relatively new form of global communication: the widespread use of the Emoji and the Emoticon by our younger business community. This powerful form of communication has many attributes that make it an enticing methodology for much of our society. Thus, it has the potential to influence business norms, societal norms and potentially legal thought and practice. To examine this new communication paradigm, we must look at how modes of business communication have changed with time and how our legal system has kept up with those changes. It is also incumbent on the law, both domestic and international, to recognize the prevailing trends in the use of Emoji and emoticons in communication and help guide countries in their use. Failure to anticipate and incorporate new modes and methods of communication would ultimately make our legal system less responsive to society's needs and

${ }^{1}$ Dr. S. B. M. Marume, E. Jaricha, T. M. Chiunye, Communication, 5 INT'L J. ENG'G SCI. INVENTION 26 (2016).

${ }^{2}$ R. Saikia, Meaning of Communication, BuS. MGMT. IDEAS, https:/ / www.businessmanagementideas.com/communication/ meaning-of-communication (last visited Feb. 6 2021). further the divide between our society and other societies who embrace and incorporate Emoji usage.

\section{COMMUNICATION}

The basic question is: what is communication's function? According to D.E. McFarland, a former fellow at the International Academy of Management:

"Communication may be broadly defined as the process of meaningful interaction among human beings. More specifically, it is a process by which meanings are perceived and understandings are reached among human beings." 1

An essential element of communication is that the communicated information can be correctly understood and is not capable of multiple meanings. ${ }^{2}$ This is a central element in all aspects of life. ${ }^{3}$ It is the potential for multiple

\footnotetext{
${ }^{3}$ Daviel Emojorho, The Role of Effective Communication in Enhancement of Library Services: An Overview of Delta State University Library, Abraka, LIBRARY PHILOSOPHY AND PRACTICE (Oct. 2010), https: / / digitalcommons.unl.edu/cgi/viewcontent.cgi?article=1 452\&context=libphilprac
} 
meanings and misinterpretations which become critical in the world of international communication.

\section{A. Which came First, the Emoji or the Emoticon?}

The emoticon predates the use of the Emoji. While it is difficult to discern who invented the Emoticon its creation is attributed to Scott E. Fahlman, a computer scientist at Carnegie Mellon University on September 19, 1982. ${ }^{4}$ The use of the emoticon was not without its critics since many people felt that good writers did not need to add such editorializing to their works. ${ }^{5}$ Nonetheless, its use took off first around Carnegie Mellon University, then to other universities in places around the world. ${ }^{6}$

The first Emojis did not appear until almost two decades later and were created by Shigetaka Kurita. ${ }^{7}$ His creation in 1999 was done as a method of condensing the character set needed to communicate via cell phone. ${ }^{8}$ He used a pictorial style of communication. 9 "The word Emoji comes from the Japanese 絵 ("e," picture), 文 ("mo," write) and 字 ("ji," character)." 10 Japanese character sets are based off of Chinese ideograms, which are a highly pictorial writing system. ${ }^{11}$ Kurita created the system as a way of overcoming early telecom giant NTT DoCoMo's email character restriction of 250 characters. ${ }^{12}$ The unique pictographs have the ability to represent longer ideas in a single image. The Emoji is truly the modern-day equivalent of a picture being worth a thousand words.

These initial communication seedlings, which were planted by two different people in two different countries, have grown into a mighty communication forest.

\section{B. What is Their Purpose?}

The initial purpose of the emoticon was to head off misreading of tone or intention in cyberspace. ${ }^{13}$ The creator of the smiley face, Dr. Scott E. Fahlman, stated that

4 Teresa N. Sneft, Emoticon, BRITANNICA, https: / / www.britannica.com/topic/emoticon (last visited Feb. 6, 2021).

5 Scott E. Fahlman, Smiley Lore :-), SCOTT E. FAHLMAN'S HOMEPAGE, https://www.cs.cmu.edu/ sef/sefSmiley.htm (last visited Apr. 12, 2021).

${ }^{6}$ Id.

${ }^{7}$ Jacob Prisco, Shigetaka Kurita: The Man Who Invented the Emoji, CNN (May 22, 2018),

https://www.cnn.com/style/article/emoji-shigetaka-kuritastandards-

manual/index.html\#: :text=When $\% 20$ Shigetaka $\% 20 \mathrm{Kurita} \% 20 \mathrm{cr}$ eated $\% 20$ the, measuring $\% 2012 \% 20$ by $\% 2012 \% 20$ pixels

${ }^{8}$ Id.

${ }^{9} \mathrm{Id}$.

${ }^{10} \mathrm{Id}$.

${ }^{11} I d$.

${ }^{12} I d$.

${ }^{13}$ Alyssa Greenberg, The evolving, unexpected power of the Emoji, $\begin{array}{lll}\text { NOVA } & \text { (SEP. 29, }\end{array}$ people's attempts at humor through the use of sarcastic comments would be misinterpreted as a serious one, thus igniting a deluge of vitriol. ${ }^{14}$ The purely text based language "[lacked] the body language or tone-of-voice cues that convey [humor or jest] when we talk in person or on the phone." 15

People suggested as a solution to the miscommunication, the inclusion of joke markers but Dr. Fahlman decided on a more simple and unique solution, the smiley / frowny face. $^{16}$

The emoticon thus became a pictorial method of displaying emotion or emotional context for otherwise plain text. If we look at the smiley face alone, there are many permutations of that face, equating to 45 more subtle emotions than simply happy or sad. ${ }^{17}$ Emoji, on the other hand, grew out of the seedling bed of the cell phone, rather than the computer-based texting of a desktop or standalone computer interface from the early 80 's. Remember, cell phone providers limited the amount of text which could be included in an email. ${ }^{18}$ With the number of cell phone users eclipsing the number of computer users, a solution to text limitation needed to be found. ${ }^{19}$ In 1999, there were approximately 492 million cell phone users worldwide compared to 248 million computer users. ${ }^{20}$ A substitute for both words and emotions that could appropriately convey the meaning intended was desperately needed.

Emojis served that dual purpose of conveying emotion as well as being a substitute for words. ${ }^{21}$ "Emojis [are now] considered a substitutive tool for non-verbal cues, ... enhanc[ing] the capacity of expressing the real meaning or the emotional intention of the message." 22

\section{Global Usage of Emoji}

When considering age brackets, "Millennials have a greater affinity for Computer-Mediated Communication,

https://www.pbs.org/wgbh/nova/article/emoji-languageculture/

${ }^{14}$ Fahlman, supra note 6.

${ }^{15} \mathrm{Id}$.

${ }^{16} \mathrm{Id}$.

${ }^{17} \mathrm{Id}$.

${ }^{18}$ Prisco, supra note 8.

${ }^{19}$ ITU, Number of mobile (cellular) subscriptions worldwide from 19932019, STATISTA (Jan. 2020), https:/ / www.statista.com/statistics/262950/global-mobilesubscriptions-since-1993/

${ }^{20} \mathrm{Id}$.

${ }^{21}$ Aleksandra Antanasova, Emojis: Why We Love Them So Much and What They Mean, SOC. MEDIA TODAY (NOV. 14, 2016), https:/ / www.socialmediatoday.com/social-networks/emojiswhy-we-love-them-so-much-and-what-they-mean-0

${ }^{22}$ Oriol J. Bosch \& Melanie Revilla, The use of emojis by Millennials, UNIVERSITAT POMPEU FABRA RECSM, July 2018, at 1, 5. 
where new communicative mechanisms as Emojis have appeared. In the United Kingdom, for instance, half of the Millennials consider that Emojis have improved the ability to interact with others. ${ }^{\prime 23}$ In evaluating Twitter alone on a global stage, "Emojis are present in nearly a quarter of the tweets in the dataset $(19.6 \%)$ and are used by well over a third of the users (37.6\%). ${ }^{\prime 24}$ It should be noted that the dataset considered only users who had greater than 100 tweets in the data collecting period. ${ }^{25}$

If we consider individual countries, it appears that developing countries lead the lists of those using Emoji the most frequently. ${ }^{26}$ The highest Emoji usage by country is as follows: Indonesia is first, followed by Paraguay, the Philippines, Argentina, Africa, Algeria and Egypt. ${ }^{27}$ If we consider Emoji usage by region, in the Arab peninsula, Qatar is first followed by the UAE. ${ }^{28}$ If we look at European Countries, Latvia is first, followed by Spain, the Czech Republic, Portugal and the Russian Federation. ${ }^{29}$

Based purely on the usage of Twitter, without considering other social media, "it appears globally that the popularity of Emojis on Twitter around the world [ is most prominent in] South-Eastern Asia and South America, while in the USA (that technically enabled the rise of Emojis) and Japan (the origin of Emojis) the usage frequency on Twitter is multiple times lower." ${ }^{30}$ These figures need context, however, because there is a difference in the number of Emojis per tweet. In the study done by Nikola Ljubesie and Darla Fiser mentioned above, "single Emojis occur in $45 \%$ of the Emoji-containing tweets, two Emojis make for 25\% of the tweets, three Emojis 15\%, four Emojis 7\%, five Emojis $3 \%$ and tweets with more than five Emojis make 5\% of all Emoji-containing tweets." ${ }^{31}$ Thus, though the number of multiple Emoji go down when we look at Emoji per single tweet, at least one in four tweets contain at least two Emoji. ${ }^{32}$ It is important to note that depending on the geographic region, a different social media may be more

\footnotetext{
${ }^{23}$ Id. at 3 .

${ }^{24}$ Nikola Ljubesie \& Darla Fiser, A Global Analysis of Emoji Usage, PROCEEDINGS OF THE 10TH WEB AS CORPUS WORKSHOP (WAC-X) AND THE EMPIRIST SHARED TASK, Aug. 2016, at 84.

${ }^{25} \mathrm{Id}$. "The highest density of tweets can easily be observed in Indonesia $(46.5 \%$ of tweets containing emojis) and the neighboring third-ranking Philippines (34.6\%). In South America the king of emojis, overall ranking second, is Paraguay (37.6\%), followed by Argentina, overall ranking sixth (30.7\%). In Africa emojis are most popular in the north, with Algeria ranking fourth (33.5\%), Egypt ranking seventh $(30.4 \%)$ and Libya ranking eight (29.7\%). In the Arab peninsula Qatar comes first (overall ranking fifth, $32.6 \%$ ), followed by UAE (ranking 10th, $27.1 \%$ ). The two highest ranking European countries are Latvia (24.4\%) and Spain (24.1\%), followed by the Czech Republic, Portugal and the Russian Federation. Interestingly, Japan, the home of emojis, is ranked $163^{\text {rd }}$ "

${ }^{26} I d$.

${ }^{27} \mathrm{Id}$.

${ }^{28} I d$.
}

popular than Twitter. ${ }^{33}$ For example, Twitter, Facebook and YouTube are banned in China, so people in China may be using Sina Weibo or other social media unique to China. $^{34}$

In the Western world, according to a study done in 2016, " $40 \%$ of all texts contained at least one Emoji, and even in countries such as Korea, one third contained these symbols." ${ }^{35}$ This study reiterates the fact that Millennials (those born after 2000) are heavy users of cell phones and Emoji. This significant fact raises the question, of whether Millennial's use of Emoji is their attempt at developing a new language or whether they are simply mimicking an ancient form of communication $?^{36}$

In the decades since their introduction in the 90s, this communication method has transformed from simply a means of expressing sarcasm or jest to communicating much more complex linguistic ideas. ${ }^{37}$ The Emoji is not just a fad. They are a means of the current generation to improve textual language by adding emotional nuance as well as a potential subtext. ${ }^{38}$

As an aside worth mentioning, linguists are currently debating whether Emoji are in fact a new language or not. There are those such as Professor Dresner from Tel Aviv University and Professor Susan Herring from Indiana University who state that Emoji do not incorporate "actual linguistic traits of English as they do not contain letters." ${ }^{\prime 39}$ These professors bring up an interesting question: "if Emojis do not have a direct convention and connection to English for example, can they really be viewed as a language?"40 The counter to their perspective is viewing the pictorial based Emoji as a more modern version of the hieroglyph, an ancient Egyptian communication modality. Regardless of the question whether the Emoji is truly a new language or simply a resurgence of another version of communication, it is clear that Emoji are widespread; as of October of 2020, the official count of recognized and

\footnotetext{
${ }^{29} \mathrm{Id}$.

${ }^{30} I d$. at 88 .

${ }^{31} \mathrm{Id}$. at 84 .

${ }^{32} \mathrm{Id}$.

${ }^{33}$ Sharath Chandra Guntuku, Mingyang Li, Louis Tay, Lyle H. Ungar, Studying Cultural Differences in Emoji Usage across the East and the West, 13 PROCEEDINGS OF THE THIRTEENTH INT'L AAAI CONFERENCE ON WEB AND SOCIAL MEDIA 226 (2019).

${ }^{34}$ Id. at 227.

${ }^{35}$ Hamza Alshenqeeti, Are Emojis Creating a New or Old Visual Language for New Generations? A Socio-semiotic Study, 7 ADVANCES IN LANGUAGE AND LITERARY STUDIES 56, 60 (2016).

${ }^{36} \mathrm{Id}$. at 56.

${ }^{37}$ Greenberg, supra note 14.

${ }^{38}$ Ariel Parades, The WIRED Guide to Emoji, WIRED (Feb. 1, 2018, 9:23 AM), https://www.wired.com/story/guide-emoji/

${ }^{39}$ Alshenqeeti, supra note 36 , at 61.

${ }^{40} \mathrm{Id}$.
} 
available Emoji was 3,521. ${ }^{41}$ 90\% of these Emoji are supported on major telecommunications platforms. ${ }^{42}$ That represents approximately 167 new Emoji created each year.

\section{Usage of Emoji by Generation}

There are differences in the use of Emoji in communication based on age that are significant, but it seems that the difference in age usage may also be colored by both geography and familiarity. "Those who have grown up with text language, mobile technologies and digital mediums are frequently more accepting of changes to the way these operate, for example incorporating Emojis into their messages." 43 Additionally, those of older generations who have been exposed to Emoji through their "work or their children/grandchildren are more accepting of changes such as increased use of Emojis." ${ }^{44}$

In Japan, women of all ages tended to use Emoji more than their male counterparts. ${ }^{45}$ It is unclear whether the fact that the Japanese keyboard "developed specifically for users to utilize the images to send non-verbal messages" ${ }^{46}$ and Emoji are a factor in their increased usage.

If we look at the United States, based on a study done by messaging technology company Holler, formally called Emoji, users under the age of 25 use Emojis in their daily communication $75 \%$ of the time. ${ }^{47}$ That number increases to $75.9 \%$ for people $25-29$ and dips lightly to $68.3 \%$ in people 30-35. ${ }^{48}$ According to an article published by Mailbird, a desktop email management client company, $61 \%$ of their respondents use Emoji in a work setting. ${ }^{49}$ Additionally, "78\% said that they enjoyed communicating with the person if they used Emojis." ${ }^{50}$ The numbers display a trend and are glimpse into the future.

The percentage of people 18-29 who use a cell phone is $96 \%{ }^{51}$ The number of people $30-49$ who use a cell phone is $92 \%$. ${ }^{52}$ Though these percentages seem similar, their impact is different. If we review the graph, (see cite below) where these figures come from, we see that there are

41 Emoji Statistics, EMOJIPEDIA, https://emojipedia.org/stats/ (last visited Apr. 12, 2021).

${ }^{42}$ Id.

${ }^{43}$ Alshenqeeti, supra note 36 , at 61.

${ }^{44}$ Id.

${ }^{45}$ Alshenqeeti, supra note 36 , at 62.

${ }^{46} \mathrm{Id}$.

${ }^{47}$ Natalie Charles, Using emojis in digital marketing copy: the dos and don'ts, COAST DigITAL (Jan. 29, 2020), https://www.coastdigital.co.uk/2020/01/29/using-emojis-indigital-marketing-copy-the-dos-donts /

${ }^{48} \mathrm{Id}$.

49 Anastasia Kryzhanovska, Business Emojis in Workplace Communications, MAILBIRD (Sep. 11, 2020), https://www.getmailbird.com/emojis-businesscommunication/

${ }^{50} \mathrm{Id}$. almost 50\% more people, ages $18-29$, using cell phones than people age 30-49. The upshot of this is that those ages 1829 who are now in the workforce or will soon be entering the business workforce will be heavy cell phone users and therefore Emoji users. There were 27.3 million college-age people in the United States in $2017 .{ }^{53}$ That translates into 26.2 million people entering the workforce for whom using Emoji as a means of communication is commonplace, if not necessary.

\section{INTERnAtional Communication, AND Business}

International business transactions are a fundamental part of our global economy. Ease and clarity of business communications coupled with appropriate level of cultural understanding are an essential element of being able to do business in a diverse and ever-changing world. The use of computers, cell phones and video conferencing in business have created electronic bridges that make speaking to someone in Beijing as simple as a call from Boston to New York.

\section{A. International Transactions in a Global Economy}

International transaction in global payment revenues totaled 1.9 trillion dollars in 2018. ${ }^{54}$ Of that figure, 0.9 trillion dollars came from the Asia-Pacific, 0.5 trillion dollars came from North America 0.3 trillion dollars came from Europe, the Middle East and Africa, and 0.3 trillion came from Latin America. ${ }^{55}$ Out of all countries, "China is the single-largest contributing country to global payments revenues, surpassing the United States by more than $\$ 100$ billion." ${ }^{56}$ It is estimated that, "nearly half of all global debit card spending now occurs in China alone." ${ }^{17}$ Further, "Mobile payments transactions grew at a 123 percent ... in China from 2013 to 2018 and, based on data published by the People's Bank of China, are estimated to have exceeded 300 billion (or roughly 210 transactions per capita) in 2018." 58 The significance here is that people globally moved from a cash society to a credit/debit card society

\footnotetext{
${ }^{51}$ Pew Research Center, Share of adults in the United States who own a smartphone from 2015-2019, by age group, STATISTA (June 2019), https://www.statista.com/statistics/489255/percentage-of-ussmartphone-owners-by-age-group/

${ }^{52} \mathrm{Id}$.

${ }^{53}$ Status and Trends in Education of Racial and Ethnic Groups, IES NCES (Feb. 2019), https://nces.ed.gov/programs/raceindicators/indicator_RAA.a sp

${ }^{54}$ Sukriti Bansal et al., Global Payments Report 2019: Amid Sustained growth, accelerating challenges demand bold actions, MCKINSEY \& COMPANY, Sep. 2019, at 1.

${ }^{55} \mathrm{Id}$. at 3.

${ }^{56} I d$. at 5 .

${ }^{57} \mathrm{Id}$.

${ }^{58} I d$.
} 
and now to one using mobile apps to conduct their transactions. ${ }^{59}$ In China, "the emergence of large ecosystem players, like Alipay and WeChat Pay, [have] boosted the growth of mobile payments" ${ }^{60}$ Society has embraced cell phone usage both as a form of communication and as a platform for conducting business transactions.

\section{B The Language of Mobile Communications in Business}

An important part of international business transactions is being able to communicate effectively. Lawyers, who specialize in contract negotiation and interpretation on an international scale, are aware of the significance of forum selection clauses, choice of law clauses, and choice of language clauses. Typically, we can assume that people from the same location will be fluent in the local language. ${ }^{61}$ Regionally, there is often a language in addition to the local language being spoken. ${ }^{62}$ For example, French is spoken in Western Africa, and Spanish is spoken in Latin America. ${ }^{63}$ But, as a general premise, "English is currently the dominant language in international commerce, [thus] laws available in English should enjoy a clear advantage over laws written in other languages." ${ }^{64}$ However, when we consider the notion of a written language, are we considering the implication, understanding, and nuance of the Emoji being used as a communication medium? I would suggest we are not. This conclusion is based on the premise that high Emoji use is currently a characteristic the young. ${ }^{65}$ The significance of that use and the subsequent normalizing of a valid method of communication may not be felt for several years. But, make no mistake it is coming. So, how does its arrival and approach affect international communication, and what must we be mindful of?

\section{What is the Purpose of Communication?}

There are multiple theories on what communication is and why we have it. From a business management perspective:

${ }^{59} \mathrm{Id}$.

${ }^{60} I d$.

${ }^{61}$ Gilles Cuiberti, The International Market for Contracts: The Most Attractive Contract Laws, 34 Nw. J. of Int'l Law \& Bus. 455, 482 (2014).

${ }^{62} \mathrm{Id}$.

${ }^{63} \mathrm{Id}$.

${ }^{64} I d$.

${ }^{65}$ ZoomInfo, 30 Emoji Statistics for Businesses, ZOOMINFO (Feb. 3, 2020), https://blog.zoominfo.com/emoji-statistics-forbusinesses/

${ }^{66}$ Saikia, supra note 3.

${ }^{67}$ Dr. S. B. M. Marume, E. Jaricha, T. M. Chiunye, Communication, 5 INT'L J. ENG'G SCI. INVENTION 26 (2016).
"Communication is a process through which an information idea or opinion is transferred to one or more number of persons. If information is communicated to only one person, it will also be termed as communication. But the essential element of communication is that the communicated information should be understood correctly and transferred in the right direction. ${ }^{\prime \prime 6}$

From an engineering perspective, seven elements are required: clarity, consistency, adequacy, timeless[ness], uniformity, flexibility and acceptability. ${ }^{67}$ Lastly, it can be said generally to be, "a process of meaningful interaction among human beings. ${ }^{168}$ It is a transfer of information in a way where the full meaning is transmitted and properly received and understood. ${ }^{69}$

By looking at these three definitions, we can cull some commonality. Communication is a process where information is transferred to another individual where the transmission's meaning is sufficiently clear and consistent such that an understanding may be reached. If we focus on communication's minimal requirements, we can agree that clarity, consistency and, given today's many modes of communication, capable of being used by the bulk of society are requisites. But do Emoji meet these? The answer depends on who you ask.

Emojis are not simply a fun method of expressing emotion but have evolved into their own language. "What adults call texting, kids call talking." 70 The language they are using is changing and evolving all the time. ${ }^{71}$ These letter combinations can range from simple combinations whose meaning might be fairly apparent such as "AMIRITE (am I right?) QQ4U (quick question for you), CWYL (chat with you later), to Emoji of a rose, cherry, pink cherry blossom, growing heart, airplane, or crown, which equate to sex trafficking. ${ }^{72}$ The assumption is that the meaning of the Emojis and the letter combinations are known to the receiver but not easily known to an onlooker.

Is the meaning of the Emoji always clear? Take the Pizza Slice Emoji. It can mean you are in the mood for pizza. It can also mean, "I love you." ${ }^{\prime 73}$ How about the Alien Face?

68 Vicky, Communication: Meaning, Purpose, Importance and Principles, YOUR ARTICLE LIBRARY, https://www.yourarticlelibrary.com/management/communica tion/communication-meaning-purpose-importance-andprinciples/60291 (last visited Apr. 12, 2021).

${ }^{69} \mathrm{Id}$.

${ }^{70}$ Toni Birdsong, Teen Texting Slang (and Emojis) Parents Should Know, MCAFEE (Apr. 6, 2019), https:/ / www.mcafee.com/blogs/consumer/teen-texting-slangand-emojis-parents-should-know /

${ }^{71} \mathrm{Id}$.

${ }^{72} I d$.

${ }^{73}$ Aneri Shah, 10 daily used emojis you never knew had a double meaning, PAPER AVOCADO (Jul. 17, 2019), 
"The Alien Emoji was created in 2010 to suggest that something is out of this world. ${ }^{\prime 74}$ Yet, in current culture, the Emoji refers to immigrants, especially those who some call illegal immigrants. ${ }^{75}$ In a short ten years, the Emoji went from saying something benign to something intended to offend.

The key to communication is transmitting a thought to another that is understood by the recipient. ${ }^{76}$ If you have had prior talks with the recipient, the probability that they understand you is greater. This conclusion rests on the assumption that if the receiver did not understand, they would eventually say so.

So, if the purpose of language is communication, and today most people ages 18-40 communicate through the use of Emoji, either predominantly or as a short-hand method of communication, how does this form of communication affect legal obligation and understanding of terms?

\section{Emoji Differences across the Globe}

Cultural and geographic differences play a significant role in Emoji understanding. ${ }^{77}$ "Emojis used to express these constructs are likely different between Eastern and Western Emoji Users: 'Insight', 'Discrepancy', 'Quantitative', 'Number', 'Time', 'Friend', and 'Work' have small or near-zero correlations."

Cultural protocol and appropriate of interpersonal conduct also have a significant effect on Emoji use and choice. $^{79}$

"East Asians and Westerners have differences in interpersonal dealings. With regard to the former, Confucianism places a premium on harmony and proper relationships as the basis for Asian society whereas Westerners often place greater importance in outcomes and direct communication (Yum 1988). This is revealed in differences in the category of 'Friend' in how Emojis are used to express that idea."

The result of this difference could lead to a Western business negotiator, not fluent in the nuance of the Eastern

https://paperavocado.com/10-daily-used-emojis-you-neverknew-had-double-meanings/

${ }^{74} \mathrm{Id}$.

${ }^{75} \mathrm{Id}$.

${ }^{76} \mathrm{Id}$.

${ }^{77}$ Guntuku, et al. supra note 34.

${ }^{78}$ Id. at 231.

${ }^{79} \mathrm{Id}$. at 231.

${ }^{80}$ Alex Rawlings, Why emoji mean different things in different cultures, BBC (Dec. 11, 2018), https://www.bbc.com/future/article/20181211-why-emojimean-different-things-in-different-cultures

${ }^{81} \mathrm{Id}$.

${ }^{82} \mathrm{Id}$.

Page 152 culture, using a given Emoji in a friendly way, intending it to lighten the mood, but instead accidentally breaching proper relationship and decorum in a way that might be viewed as an insult.

There are also icon differences based on geographic location. The thumbs-up Emoji is a symbol of approval in the United States but, "traditionally in Greece and the Middle East it has been interpreted as vulgar and even offensive." ${ }^{80}$ In the U.S. or other Western cultures, the angel Emoji, which might be seen as implying innocence, purity, perfection or having performed a good deed, has a completely opposite interpretation in China as "a sign for death, and may be perceived as threatening." ${ }^{81}$ Also in China, the applause Emoji, which is the Western equivalent of good job or congratulations, equates to making love. $^{82}$

Cultural interpretations aside, what does a thumbs up Emoji mean? Consider two account representatives negotiating an international contract worth 10 million dollars. They have been stuck on a hold back figure for weeks. It is the last term needed to finish the negotiation. Finally, one party sends a text message suggesting a 1million-dollar holdback, and the other party texts back thumbs up ( ). Does it signify good job, I agree, or nice suggestion? Do we have an agreement or not?

\section{E. A Legal Black Hole}

There are several significant pieces of legislation that affect American business interests as they pertain to Emoji use in international contracts. One is Title 15 U.S. Code SUBCHAPTER I-ELECTRONIC RECORDS AND SIGNATURES IN COMMERCE, called the Electronic Signatures in Global Commerce and National Commerce Act, which was signed into Law by President Clinton in 2000. "Esign," as it is known, does not change the law within its jurisdictional scope other than to broaden the medium of execution and exchange of information. ${ }^{83}$ That which was previously executed on paper may now use an electronic or digital form as the medium. ${ }^{84}$ It also recognizes that a signature may be done by electronic

\footnotetext{
${ }^{83}$ Margo H.K. Tank, David Whitaker, Andrew Grant, A short primer on applicable US eSignature laws, DLA PIPER (May 2, 2018), https://www.dlapiper.com/en/us/insights/publications/2018 L07/esignature-and-epay-news-and-trends-july-2018/a-shortprimer-on-applicable-us-esignature-laws /

${ }^{84} 15$ U.S.C. $\S 7001$
}

(a)IN GENERAL Notwithstanding any statute, regulation, or other rule of law (other than this subchapter and subchapter II), with respect to any transaction in or affecting interstate or foreign commerce-

(1) a signature, contract, or other record relating to such transaction may not be denied legal effect, validity, or enforceability solely because it is in electronic form; and

American Jaurnal of Trade and Policy • Vol $8 \bullet$ Issue 2/2021 
means. ${ }^{85}$ "The term "electronic signature" means an electronic sound, symbol, or process, attached to or logically associated with a contract or other record and executed or adopted by a person with the intent to sign the record." ${ }^{86}$ Nowhere in Esign does it specifically mention the use of an Emoji, though it does state that an electronic signature can be done via the use of a symbol.

Another significant piece of legislation pertaining to Emojis is the UETA. This law "was approved and recommended by the National Conference of Commissioners on Uniform State Laws in July 1999." 87 UETA has been adopted in all the states except three, though much like the Uniform Commercial Code, each state has adopted its own law governing signatures and records. ${ }^{88}$ Under the UETA, "“Electronic signature" means an electronic sound, symbol, or process attached to or logically associated with a record and executed or adopted by a person with the intent to sign the record." ${ }^{89}$

On an international scale, there is the United Nations Convention on the Use of Electronic Communications in International Contracts. " "This Convention applies to the use of electronic communications in connection with the formation or performance of a contract." ${ }^{\prime 91}$ Under Article 8 of the Convention: "A communication or a contract shall not be denied validity or enforceability on the sole ground that it is in the form of an electronic communication."92 The convention also seems to allow for the use of Emoji as a means of indicating acceptance of contractual terms, though not specifically. ${ }^{93}$

It is this lack of acknowledgement of the Emoji and its impact on communication as well as the generality of the language used in both the US statutes and the International Convention which leads us over a precipice of uncertainty. It is my position that we must acknowledge that the use of Emoji has become more than a passing fad among our younger business professionals. That acceptance mandates that we act in a way to strengthen and legitimize its acceptance and use. My proposal for this evolution is below.

(2)a contract relating to such transaction may not be denied legal effect, validity, or enforceability solely because an electronic signature or electronic record was used in its formation.

${ }^{85} 15$ U.S.C. $\S 7006(5)$

${ }^{86} \mathrm{Id}$.

${ }^{87} \mathrm{Id}$.

${ }^{88} \mathrm{Id}$.

${ }^{89} \mathrm{Id}$.

${ }^{90}$ UNCITRAL, United Nations Convention on the Use of Electronic Communications in International Contracts, WORLD INTELLECTUAL PROPERTY ORGANIZATION, https://www.wipo.int/edocs/lexdocs/treaties/en/uncitraluecic/trt uncitral uecic.pdf (last visited Apr. 12, 2021).

${ }^{91} \mathrm{Id}$. at 2.

\section{CONCLUSION AND SOLUTION}

I would suggest that in addition to including choice of language, choice of law and forum selection in international contracts, we should also include a new item called "choice of symbol." Currently, the Unicode Consortium, a non-profit organization whose members include most major cellular carriers and software companies, administers what Emoji are going to be available to the public at large. I suggest that the Consortium add to their character set not only the code for producing the Emoji but also its accepted definition. By doing this, at least US companies could reference those definitions when creating and or negotiating contracts.

It would also be beneficial if the international community could in some way define or at least combine the creation of Emoji cross-culturally. At present, China, as an example, regulates their own Emoji usage, but it would seem that if some sort of rudimentary set of Emoji were placed in the UN Convention on Electronic Communication, at least we would have a starting point from which to form some type of database. As new Emoji are created in varying countries, their creation code, picture, and intended meaning could be listed in the UN database, thus adding clarity to this new form of communication and enhancing communication internationally and cross-culturally. Our failure to recognize and deal with this new communications medium will result in their being more and more contractual uncertainty. This uncertainty will further result in additional global law suits and a reduction in trade certainty. This is not a passing fad but a force to be reckoned with driven by our future millennial leaders and business executives.

$$
--0--
$$

92 Id. at 5.

${ }^{93}$ Id. at 5: "Where the law requires that a communication or a contract should be signed by a party, or provides consequences for the absence of a signature, that requirement is met in relation to an electronic communication if:

(a) A method is used to identify the party and to indicate that party's intention in respect of the information contained in the electronic communication; and

(b) The method used is either:

(i) As reliable as appropriate for the purpose for which the electronic communication was generated or communicated, in the light of all the circumstances, including any relevant agreement; or

(ii) Proven in fact to have fulfilled the functions described in subparagraph (a) above, by itself or together with further evidence." 


\section{Acknowledgement}

The author wishes to thank Amanda Shoemaker for her research assistance and Bentley University for the research support. 